\title{
Alteraciones bucales asociadas a diabetes mellitus tipo 1 en niños y adolescentes
}

\section{Buccal alterations associated to type 1 diabetes mellitus in children and adolescents}

\author{
Yaimeé Guerrero-Díaz*
}

Facultad de Ciencias Médicas, Departamento de Estomatología General Integral, Universidad de Ciencias Médicas de Holguín Mariana Grajales Coello, Holguín, Cuba

\section{RESUMEN}

Introducción: La diabetes mellitus tipo 1 (DM1) es una enfermedad sistémica crónica con repercusión bucal. La incidencia de DM1 en los niños menores de 15 años de edad ha aumentado en un 2-5\% por año. Objetivo: Determinar la asociación de las alteraciones bucales y la DM1 en niños y adolescentes con esta patología. Material y métodos: Se realizó un estudio observacional de casos y controles, con un grupo de niños y adolescentes con DM1 (grupo 1), de edades comprendidas entre 3 a 18 años, y un grupo de niños y adolescentes sin DM (grupo 2) de edades y sexo comparables. Resultados: Los pacientes con diabetes resultaron más afectados por caries dental (64.44\%), úlceras (44.44\%) y periodontopatías (86.67\%) que los pacientes sin diabetes, siendo estos resultados significativos estadísticamente, a diferencia del comportamiento de la higiene bucal, donde las diferencias no fueron estadísticamente significativas. Conclusiones: La DM 1 se relaciona con las alteraciones bucales. Así, los niños y adolescentes que padecen diabetes presentan mayor frecuencia de caries dental, periodontopatías y úlceras que los niños y adolescentes sin diabetes; no sucede del mismo modo con la higiene bucal.

Palabras clave: Diabetes tipo 1. Alteraciones bucales. Niños. Adolescentes.

\section{ABSTRACT}

Background: Diabetes mellitus type 1 (DM1) is a chronic systemic disease with repercussions at the oral level. The incidence of DM1 in children under 15 years of age has increased by $2-5 \%$ per year. Objective: To determine the association of oral disorders and DM1 in children and adolescents with this pathology. Material and methods: An observational study and controls of cases was carried out with a group of children and adolescents aged between 3 to 18 years with DM1 (Group 1) and a group of children and adolescents with comparable ages and sex without DM (Group 2). Results: Patients with diabetes were more affected by dental caries (64.44\%), ulcers (44.44\%), and periodontal diseases $(86.67 \%)$ than patients without diabetes. These have been statistically significant results, unlike the behavior of oral hygiene where the differences were not statistically significant. Conclusions: DM1 is related to oral disorders, so diabetic children and adolescents have a higher frequency of dental caries, periodontal disease, and ulcers than children and adolescents without diabetes. This is not the case with oral hygiene.

Key words: Diabetes type 1. Oral disorders. Children. Adolescents.
Disponible en internet: 17-03-2021 Rev ALAD. 2021;11:1-6

2248-6518 / @ 2021 Asociación Latinoamericana de Diabetes. Publicado por Permanyer. Este es un artículo open access bajo la licencia CC BY-NC-ND (http://creativecommons.org/licenses/by-nc-nd/4.0/). 


\section{INTRODUCCIÓN}

El comportamiento de la diabetes mellitus (DM) en las últimas tres décadas ha mostrado características que muestran a esta enfermedad como un verdadero problema mundial de salud pública. Inicialmente más marcado en países desarrollados, en la actualidad se ha confirmado el aumento de su incidencia en jóvenes tanto en países desarrollados como en vías de desarrollo'.

De acuerdo con la Asociación Americana de la Diabetes (ADA) y el Programa Nacional para la Educación sobre la Diabetes, alrededor de 208,000 personas menores de 20 años padecen esta enfermedad, en su mayoría de tipo $1\left(\mathrm{DM}_{1}\right)^{2}$. Sin embargo, la diabetes tipo 2, una enfermedad que solía observarse principalmente en adultos mayores de 45 años, es cada vez más común entre los jóvenes. Esto se debe principalmente al aumento de las tasas de obesidad en niños y adolescentes ${ }^{3}$.

La diabetes cobra especial interés por ser una de las enfermedades metabólicas crónicas más frecuentes en la infancia. Los pacientes con esta patología tienen mayor riesgo de desarrollar otras enfermedades que deterioran progresivamente su calidad de vida. Estas complicaciones se encuentran con mayor frecuencia en pacientes descompensados ${ }^{4}$.

Entre las alteraciones bucales más frecuentes en estos pacientes se encuentran las elevadas concentraciones de glucosa sanguínea de manera sostenida, que generan disminución del flujo salival, lo que contribuye a la acumulación de placa dentobacteriana y a la formación de tártaro, al desarrollo de caries dental, halitosis y enfermedad periodontal. Los niños y adolescentes con DM1 suelen presentar, en términos generales, mayor respuesta de los tejidos periodontales, lo que se expresa en mayor inflamación gingival y mayor predisposición a padecer enfermedades periodontales graves ${ }^{5}$.
Resulta preocupante el impacto de la diabetes en los tejidos bucales, sin embargo, las afectaciones bucales han sido poco estudiadas en edades pediátricas. Por ello el objetivo del presente trabajo es determinar la asociación de la DM1 y las alteraciones bucales en niños y adolescentes con esta patología.

Esta investigación favorece un acercamiento del equipo básico de trabajo (médico, enfermera y estomatólogo) al paciente y su familia, para poder prevenir y tratar de forma integral las alteraciones presentes en la cavidad bucal.

\section{OBJETIVO}

Determinar la asociación entre la DM1 y las alteraciones bucales en niños y adolescentes con esta patología.

\section{MATERIAL Y MÉTODOS}

Se realizó un estudio observacional de casos y controles, en un grupo de niños y adolescentes con DM (grupo 1) en las edades comprendidas entre 3 y 18 años, con el objetivo de determinar la asociación entre la DM1 y las alteraciones bucales en niños y adolescentes con esta patología, en el periodo comprendido entre marzo y diciembre del 2019.

Como criterios de inclusión para el grupo 1 se adoptaron que todos fueran personas con DM1, clasificados según el criterio de la $\mathrm{ADA}^{2}$, que pertenecieran al grupo de edades antes señaladas y que sus padres o tutores dieran su aprobación para la participación en este estudio.

También se incluyó un grupo de niños y adolescentes sin DM1, de edades y sexo comparables, procedentes del Área de Salud de la Clínica Estomatológica 
Mario Pozo Ochoa, Municipio Holguín, previa autorización de sus padres, conformando de esta forma el grupo 2.

Se excluyeron del estudio aquellos pacientes cuyos padres no dieron el consentimiento de participación y pacientes portadores de otra enfermedad sistémica cuyo tratamiento provocaba el agrandamiento gingival.

Se seleccionó la misma cantidad de pacientes del grupo 1 (45 pacientes) y del grupo 2 (45 pacientes), por lo que la muestra incluyó un total de 90 niños y adolescentes. Todos los incluidos en grupo 1 procedieron de la Consulta de Endocrinología Pediátrica del Hospital Pediátrico de la provincia de Holguín. Se incluyeron de forma consecutiva durante un periodo de nueve meses.

\section{Obtención de la información y variables}

A todas las personas incluidas en el estudio se las entrevistó y se plasmaron en una planilla de recolección de datos todos los aspectos de interés; las entrevistas y exámenes bucales se realizaron siempre por la misma estomatóloga. Se precisó en todos los casos las siguientes variables:

- Edad: 3-6, 7-10, 11-14 y 15-18 años según años cumplidos.

- Sexo: masculino o femenino, según sexo biológico.

- Presencia o ausencia de úlceras según examen bucal.

- Caries dental: se determinó el índice de COP-D (cariados, obturados y perdidos) para cuantificar la prevalencia de la caries dental.
- Enfermedad periodontal: se emplearon los criterios del índice periodontal de Russell. Este índice es capaz de medir tanto la inflamación gingival como el grado de destrucción ósea y determina la prevalencia y gravedad de las periodontopatías de una población dada (no se incluyeron los criterios radiográficos). Criterio según puntuación del Índice de Russell:

- o: encía sana, sin evidencia de alteración.

- 1: gingivitis leve, área de inflamación en la encía libre, no circunscribe al diente.

- 2: gingivitis, área de inflamación en toda encía libre que rodea al diente, pero no existe ruptura evidente de la adherencia epitelial.

- 6: gingivitis con formación de bolsa (ruptura de la adherencia epitelial y evidencia de bolsa periodontal). No hay interferencia con la masticación, ni existe movilidad dentaria.

- 8: destrucción avanzada con pérdida de la función masticatoria, bolsa profunda y movilidad evidente del diente ${ }^{6}$.

- Higiene bucal: se utilizó un método para medir la placa dentobacteriana sobre la base del índice de análisis de higiene bucal de Love, se clasificó como buena hasta el 20\% de placa dentobacteriana y como mala $>20 \%$ de placa dentobacteriana 7 .

\section{Técnicas y procedimientos de análisis estadístico}

Los resultados se llevaron a tablas y cuadros de interés estadístico. El análisis estadístico se realizó mediante cálculo de distribución de frecuencia simple (porcentaje). 
Se aplicó la prueba de chi cuadrada para evaluar la asociación entre las variables con un $a=0.01$. En algunos resultados el valor de p se escogió teniendo en cuenta la corrección de Yates.

\section{Ética médica}

El estudio se realizó acorde a los principios de la ética médica y la Declaración de Helsinki, lo cual quedó plasmado en el consentimiento informado. Se brindó información a los pacientes y sus tutores sobre los objetivos, métodos y beneficios de la intervención, y se les garantizó el anonimato de los resultados. Ellos autorizaron su participación en la investigación, pudiendo abandonarla en el momento en que lo deseasen. Este consentimiento informado por parte de padres y tutores se realizó por escrito. En este estudio, además, se pidió autorización mediante la firma de un autorizo a la dirección del centro donde se realizó la investigación para desarrollar estas actividades.

\section{RESULTADOS}

En este estudio predominó el sexo femenino, con un $58.9 \%$ y el grupo de edad de $15-18$ años con un $30.1 \%$ en el grupo de estudio (resultado similar en el grupo 2).

Como se observa en la tabla 1 , el $64.44 \%$ del grupo 1 presentaba caries dental, a diferencia del grupo 2, con un $33.33 \%$, con asociación estadísticamente significativa $(p=0.0007)$.

La tabla 2 muestra que el $37.78 \%$ del grupo 1 y el $28.89 \%$ del grupo 2 presentan una higiene bucal deficiente, una diferencia que no es estadísticamente significativa ( $p=0.1473$ ).
TABLA 1. Distribución de los grupos según afectación por caries dental

\begin{tabular}{|l|c|c|c|c|c|c|}
\hline \multirow{2}{*}{ Grupos } & \multicolumn{4}{|c|}{ Caries dental } & \multicolumn{2}{c|}{ Total } \\
\cline { 2 - 7 } & \multicolumn{2}{|c|}{ Sí } & \multicolumn{2}{c|}{ No } & \multicolumn{2}{|c|}{} \\
\cline { 2 - 7 } & No & $\%$ & No & $\%$ & No & $\%$ \\
\hline Grupo 1 & 29 & 64.44 & 16 & 35.56 & 45 & 50 \\
\hline Grupo 2 & 15 & 33.33 & 30 & 66.67 & 45 & 50 \\
\hline Total & 44 & 48.89 & 46 & 51.11 & 90 & 100 \\
\hline & & & $\chi^{2}=11.4286$ & \multicolumn{2}{c|}{$p=0.0007$} \\
\hline
\end{tabular}

TABLA 2. Distribución de los grupos según afectación por higiene bucal

\begin{tabular}{|l|c|c|c|c|c|c|}
\hline \multirow{2}{*}{ Grupos } & \multicolumn{4}{|c|}{ Higiene bucal } & \multicolumn{2}{c|}{ Total } \\
\cline { 2 - 7 } & \multicolumn{2}{|c|}{ Eficiente } & \multicolumn{2}{c|}{ Deficiente } & \multicolumn{2}{c|}{} \\
\cline { 2 - 7 } & No & $\%$ & No & $\%$ & No & $\%$ \\
\hline Grupo 1 & 28 & 62.22 & 17 & 37.78 & 45 & 50 \\
\hline Grupo 2 & 32 & 71.11 & 13 & 28.89 & 45 & 50 \\
\hline Total & 60 & 66.67 & 30 & 33.33 & 90 & 100 \\
\hline & & & $\chi^{2}=2.1053$ & \multicolumn{2}{c|}{$p=0.1473$} \\
\hline
\end{tabular}

TABLA 3. Distribución de los grupos según afectación por presencia de úlceras

\begin{tabular}{|l|c|c|c|c|c|c|}
\hline \multirow{2}{*}{ Grupos } & \multicolumn{3}{|c|}{ Presencia de úlceras } & \multicolumn{2}{c|}{ Total } \\
\cline { 2 - 6 } & \multicolumn{2}{|c|}{ Sí } & \multicolumn{2}{c|}{ No } & \multicolumn{2}{c|}{} \\
\cline { 2 - 6 } & No & $\%$ & No & $\%$ & No & $\%$ \\
\hline Grupo 1 & 20 & 44.44 & 25 & 55.56 & 45 & 50 \\
\hline Grupo 2 & 5 & 11.11 & 40 & 88.89 & 45 & 50 \\
\hline Total & 25 & 27.78 & 65 & 72.22 & 90 & 100 \\
\hline & & & $\chi^{2}=12.7366$ & $p=0.0002$ \\
\hline
\end{tabular}

En cuanto a la presencia de úlceras, como se ve en la tabla 3 , se encontraron en el $44.44 \%$ del grupo 1 y solo en el $11.11 \%$ del grupo 2 , diferencia que resultó estadísticamente significativa $(p=0.0002)$.

La tabla 4 indica una asociación estadísticamente significativa $(p=0.0006)$ en cuanto a la presencia 
TABLA 4. Distribución de los grupos según afectación por enfermedad periodontal

\begin{tabular}{|l|c|c|c|c|c|c|}
\hline \multirow{2}{*}{ Grupos } & \multicolumn{3}{|c|}{ Enfermedad periodontal } & \multicolumn{2}{c|}{ Total } \\
\cline { 2 - 6 } & \multicolumn{2}{|c|}{ Sí } & \multicolumn{2}{c|}{ No } & \multicolumn{2}{c|}{} \\
\cline { 2 - 6 } & No & $\%$ & No & $\%$ & No & $\%$ \\
\hline Grupo 1 & 39 & 86.67 & 6 & 13.33 & 45 & 50 \\
\hline Grupo 2 & 18 & 40 & 27 & 60 & 45 & 50 \\
\hline Total & 57 & 63.33 & 33 & 36.67 & 90 & 100 \\
\hline & & & \multicolumn{2}{|c|}{$\chi^{2}=11.3374$} & \multicolumn{2}{c|}{$p=0.0006$} \\
\hline
\end{tabular}

de afectaciones periodontales, presentes en el $86.67 \%$ del grupo $1 \mathrm{y}$ en el $13.33 \%$ del grupo 2 .

\section{DISCUSIÓN}

La relación entre la DM y las manifestaciones bucales es algo controvertida entre los diferentes autores, pues aun cuando se observa mayor incidencia de estas patologías en algunos estudios, también se afirma que un paciente con diabetes controlado metabólicamente responde de forma similar o igual que uno sano.

En el caso de la caries dental, este estudio presenta una asociación estadísticamente significativa, coincidiendo con el trabajo de Alavi, et al. ${ }^{8}$ realizado en Irán, en la Universidad de Ciencias Médicas de Shiraz, donde se estudiaron 50 pacientes (22 niños y 28 niñas) con DM1 metabólicamente bien controlada y les midieron el índice COPD (dientes cariados, obturados y perdidos) con radiografías y con examen clínico. Los resultados fueron comparados con los obtenidos en un grupo de escolares de control. El índice COPD en los pacientes con diabetes fue más alto que en el grupo control, lo que los autores atribuyen a la reducción de secreción salival en los enfermos diabéticos ${ }^{8}$.

Difiere del estudio realizado en Turquía, Orbak, et al. ${ }^{9}$, quienes controlaron a 50 niños con DM1 y los compararon con otros 50 que no presentaban esta patología. Ellos querían investigar la influencia de la DM1 en la dentición y en la salud oral. En relación con las caries, sus resultados revelaron que los niños con DM1 tenían menos caries que los controles sin la enfermedad, en todas las edades ${ }^{9}$. En lo referente a la higiene bucal, los resultados de este estudio no fueron significativos desde el punto de vista estadístico, aunque se reconoce que la disminución del flujo salival puede incidir de forma negativa en la autolimpieza de la cavidad oral, lo cual no concuerda con Miranda, et al. ${ }^{10}$, donde la afectación de la higiene bucal fue estadísticamente significativa en los pacientes que padecen DM con respecto al grupo de pacientes no diabéticos ${ }^{10}$.

Una higiene bucal deficiente constituye el inicio de una enfermedad periodontal, patología que ha sido fuertemente asociada a la DM. Los mecanismos potenciales por los que se presenta esta enfermedad son la resistencia disminuida a la infección, flora bacteriana bucal alterada, cambios vasculares y metabolismo anormal del colágeno ${ }^{11}$. La gravedad y extensión de la periodontitis en el paciente diabético parece estar relacionada con el control de la diabetes. En el paciente con DM que se encuentra bien controlado metabólicamente se da una respuesta tisular normal y una defensa normal contra las infecciones ${ }^{12}$. Este estudio tiene como resultado una asociación significativa entre estos aspectos, coincidiendo con el trabajo de Lalla, et al. ${ }^{13}$, que estudiaron la presencia de caries y enfermedad periodontal en 182 niños y adolescentes de entre 6 y 18 años con diabetes, comparándolos con 160 sujetos semejantes, pero sin la enfermedad. Encontraron que los niños con DM tenían más placa dental y más inflamación gingival que los controles que no padecen diabetes. La enfermedad periodontal también era significativamente mayor en los niños con DM y los resultados del estudio sugerían que la destrucción periodontal puede comenzar muy temprano en la 
vida del paciente con esta patología y hacerse más acentuada ${ }^{13}$.

Coincide además con Arheiam y Omar ${ }^{14}$, donde se evidencia el predominio de la afectación y la necesidad de tratamiento periodontal en los niños con diabetes ${ }^{14}$.

Las aftas bucales tienen una etiología variada y algunos estudios, como el de Jiménez-Echemendia, et al. ${ }^{15}$, plantean que las úlceras predominan en aquellos que padecen de DM con respecto a los que no ${ }^{15}$. Esto coincide con los resultados obtenidos en este estudio.

\section{CONCLUSIONES}

La DM1 presenta una relación significativa con las alteraciones bucales. Los niños y adolescentes con esta patología presentan mayor frecuencia de caries dental, periodontopatías y úlceras si se compara con los niños y adolescentes que no la padecen; en cuanto a la higiene bucal, la asociación no es significativa.

\section{CONFLICTO DE INTERESES}

La autora declara no tener conflicto de intereses.

\section{FINANCIAMIENTO}

La presente investigación no ha recibido ninguna beca específica de agencias de sectores públicos, comercial o sin ánimo de lucro.

\section{RESPONSABILIDADES ÉTICAS}

Protección de personas y animales. Los autores declaran que para esta investigación no se han realizado experimentos en seres humanos ni en animales.
Confidencialidad de los datos. Los autores declaran que han seguido los protocolos de su centro de trabajo sobre la publicación de datos de pacientes.

\section{Derecho a la privacidad y consentimiento informado.} Los autores han obtenido el consentimiento informado de los pacientes y/o sujetos referidos en el artículo. Este documento obra en poder del autor de correspondencia.

\section{BIBLIOGRAFÍA}

1. Ledón Llanes L. La comunicación en el campo de la diabetes mellitus, algunas pautas para su acción efectiva. Rev Cubana Endocrinol. 2014; 25(2):124-38.

2. American Diabetes Association. Standards of medical care in diabetes-2013. Diabetes Care. 2013;36(Suppl 1):s11-s60.

3. Arrieta BJJ, Bartolomé VB, Jiménez ME, Saavedra VP, Arrieta BFJ. Problemas bucodentales en pacientes con diabetes mellitus (I): Indice de placa y caries dental. Med Oral. 2013;8:97-109.

4. Ministerio de Salud Pública de Cuba. Dirección de Registros Médicos y Estadísticas de Salud. Anuario Estadístico de Salud, 2018. 46 edición [Internet]. La Habana: MINSAP; 2019. Disponible en: files.sld.cu/dne/ files/2018/04/Anuario-Electronico-Español-2017-ed-2018.pdf

5. López del Valle LM, Ocasio LC. Comparing the oral health status of diabetic and non-diabetic children from Puerto Rico: a case-control pilot study. P R Health Sci J [Internet]. 2011 Sep [Consultado: 23 de octubre 2019]. Disponible en: http://www.ncbi.nlm.nih.gov/pubmed/21932712.

6. Pozo-Noguera CA, Balseca-lbarra MC. Enfermedad periodontal en pacientes diabéticos. Dominio de las Ciencias. 2017;3(2):963-90.

7. Bascones Martínez A, Muñoz Corcuera M, Bascones Ilundain J. Diabetes y periodontitis: una relación bidireccional. Med Clin. 2015;145(1):31-5.

8. Alavi AA, Amirhakimi E, Karami B: The prevalence of dental caries in 5-18-year-old insulin-dependent dia-betics of Fars Province, Southern Iran. Arch Iran Med. 2016;9:254-60.

9. Orbak R, Simsek S, Orbak Z, Kavrut F, Colak M. The influence of type1 diabetes mellitus on dentition and oral health in children and adolescents. Yonsei Med J. 2008 Jun 30;49(3):357-65. doi: 10.3349/ymj. 2008.49.3.357. PMID: 18581583; PMCID: PMC2615350.

10. Miranda OX, Troncoso PJ, Rodríguez SC, Aravena TP, Jiménez del RP. Caries e índice de higiene oral en niños con diabetes mellitus tipo 1. Rev Chil Ped. 2013;84(5):527-31.

11. Morales Hernández L, Contreras Álvarez M, Rodríguez Tizcareño H. Tratamiento implantológico en paciente con periodontitis agresiva asociada a diabetes mellitus. Reporte de caso clínico. Rev Odont Mex. 2016;20(1):33-8.

12. Egito V, Novaes $M$, Luchéis S FA, Albuquerque MF AW, Santana CL. Prevalence of oral mucosa lesions in diabetic patients: a preliminary study. Rev Bras Otorri. 2018;74(3):423-8.

13. Lalla E, Cheng B, Lal S, Tucker S, Greenberg E, Goland R, et al.: Periodontal changes in children and adolescents with diabetes: A case-control study. Diabetes Care. 2006;29:295-9.

14. Arheiam A, Omar S. Dental caries experience and periodontal treatment needs of 10- to 15-year old children with type 1 diabetes mellitus. Int Dent J. 2014;64(3):150-4.

15. Jiménez-ET, Nadal-Gutiérrez G, Licea PM. Salud bucal en adultos con diabetes tipo 1 asistentes al Centro de Atención al Diabético. La Habana. Rev Perú Epid. 2014;18(1):1-7. 